\title{
Diagnostic value of fluorine-18 fluorodeoxyglucose positron emission tomography/computed tomography in sublingual and submandibular salivary gland tumors
}

\author{
SIRUI MA $^{1}$ and YIYAN LIU ${ }^{2}$ \\ ${ }^{1}$ Division of General Surgery, Department of Surgery; ${ }^{2}$ Division of Nuclear Medicine, Department of Radiology, \\ New Jersey Medical School, Rutgers University, Newark, NJ 07103, USA
}

Received November 18, 2019; Accepted April 22, 2020

DOI: $10.3892 / \mathrm{mco} .2020 .2097$

\begin{abstract}
The aim of the present study was to compare the diagnostic accuracy of fluorine-18 fluorodeoxyglucose positron emission tomography/computed tomography (F-18 FDG $\mathrm{PET} / \mathrm{CT}$ ) with that of conventional imaging studies (CIS), such as CT or magnetic resonance imaging (MRI), in the clinical diagnosis and staging of submandibular and sublingual salivary gland tumors. In addition, the data obtained were used to evaluate the significance of maximum standardized uptake value (SUVmax) in diagnosing benign or malignant lesions. For the present study, 18 patients with submandibular or sublingual neoplasms underwent F-18 FDG PET/CT imaging with accompanying CT or MRI. The diagnostic values from 43 F-18 FDG PET/CT scans and 28 CIS of the 18 patients were compared to the gold standard histopathological and/or cytopathological diagnosis. The results demonstrated that the diagnostic accuracy for predicting primary tumors was similar between F-18 FDG PET/CT and CIS. By contrast, PET/CT imaging was more accurate in detecting lymph node metastasis compared with CT or MRI (95.4 vs. 66.7\%). F-18 FDG PET/CT had a sensitivity of $88.9 \%$ and a specificity of $97.1 \%$, whereas CT or MRI had a sensitivity of $54.5 \%$ and a specificity of $75.0 \%$. F-18 FDG PET/CT also enabled screening for distant metastasis, as observed in 10 cases in the present study. Furthermore, there were no significant differences in SUVmax between benign or malignant salivary gland lesions, as high glucose metabolism was also observed in benign tumors. In conclusion, F-18 FDG PET/CT provides more accurate diagnostic information for the evaluation of submandibular and sublingual salivary gland tumors as compared with CIS in terms of lymph node and distant metastasis.
\end{abstract}

Correspondence to: Dr Sirui Ma, Division of General Surgery, Department of Surgery, New Jersey Medical School, Rutgers University, 185 S Orange Avenue, Newark, NJ 07103, USA

E-mail: siruima@njms.rutgers.edu

Key words: positron emission tomography/computed tomography, oral cancer, head and neck cancer, accuracy, salivary gland

\section{Introduction}

Salivary gland tumors are relatively rare neoplasms that comprise $6-8 \%$ of all head and neck cancers (1). These tumors are a widely heterogeneous group that differ in their anatomic site of origin, histology and clinical presentation. The parotid gland is the most frequent site of salivary gland tumors, accounting for $80-85 \%$ of the cases (2). Of parotid gland tumors, $70-85 \%$ are benign (2). Salivary gland tumors originate less frequently in the submandibular, sublingual and minor salivary glands (1). Furthermore, $40-45 \%$ of submandibular gland tumors and $70-90 \%$ of sublingual gland tumors are malignant (1). Salivary gland tumors are associated with a high mortality rate, which may be attributed by the fact that they are more prone to metastasize to distant sites compared with other head and neck cancers (2). The rate of metastasis is $20-28 \%$ for submandibular and $>90 \%$ for sublingual gland tumors (2-4). It has been reported that regional and distant metastasis of salivary gland cancer are important prognostic indicators for survival post-treatment (5).

Conventional imaging studies (CIS), such as computed tomography (CT) and magnetic resonance imaging (MRI), have been utilized to distinguish between benign and malignant lesions, but their results have been inconsistent (6-8). Fluorine-18 fluorodeoxyglucose positron emission tomography (F-18 FDG PET)/CT imaging has been gaining more recognition for having the potential to characterize neoplasms at the initial evaluation, as well as to reassess salivary gland tumors during subsequent follow-up visits (9). CT is useful for identifying salivary gland lesions and calcifications within the lesions. However, the value of CT in defining tumor extent and differentiating neoplasms is limited due to its relatively poor soft tissue contrast (10). MRI, on the other hand, is better for tumor characterization. Its higher soft tissue resolution allows for better identification of the internal tumor characteristics, better definition of its size and margins, and determination of the presence of regional metastasis (10). However, MRI is costlier and its value in evaluating a large area of the body for metastasis is limited $(11,12)$. In comparison, F-18 FDG PET/CT is a non-invasive imaging modality that offers unique information on neoplasms regarding their metabolic activity, anatomic localization and possible distant metastasis by scanning the whole body. 
Accurate TNM staging is important for the prognosis of submandibular and/or sublingual salivary gland cancers $(3,5,13)$. However, F-18 FDG-PET/CT is not yet included in recent National Comprehensive Cancer Network guidelines due to the rarity of these high-risk salivary gland neoplasms (14). To the best of our knowledge, there have yet been no studies on the diagnostic accuracy of F-18 FDG PET/CT specifically for submandibular and sublingual gland tumors (15-16). Therefore, the aim of the present study was to uncover the role of F-18 FDG PET/CT in the management of submandibular and sublingual gland tumors compared with concurrent CIS, such as CT or MRI.

\section{Patients and methods}

Ethics approval and patient selection. Approval for this retrospective study was obtained from the Institutional Review Board. Patients were identified through a search of F-18 FDG PET/CT dictation reports stored in the University Hospital database at the Advanced Imaging Center in University Hospital (Newark, USA) between 01/01/2010 and 06/30/2018. Patients who underwent a preoperative F-18 FDG PET/CT for submandibular or sublingual salivary gland tumors with comparative CT or MRI were selected. In addition, 4 patients with postoperative F-18 FDG PET/CT scans were included with comparative CT or MRI in subsequent follow-up evaluations.

Subjects. A total of 18 patients with either submandibular or sublingual salivary gland tumors were selected from the University Hospital database between January 2010 and June 2018. From the 18 subjects included in the analysis, a total of 43 F-18 FDG PET/CT scans with contrast-enhanced CT or MRI were obtained for this study (Table I). A total of 14 F-18 FDG PET/CT imaging studies were performed for initial diagnosis and cancer staging, while the remaining 29 imaging studies were performed to restage the disease and evaluate recurrence during the follow-up period. The 29 restaging F-18 FDG PET/CT scans were performed on 8 patients.

The histopathological diagnoses included 5 mucoepidermoid carcinomas, 3 adenoid cystic carcinomas, 3 lymphomas, 1 squamous cell carcinoma, 1 basal cell adenocarcinoma, 1 submandibular duct carcinoma, 1 synovial cell sarcoma, 2 pleomorphic adenomas and 1 lymphoid hyperplasia. The mean age of the patients was 58.1 years, with a standard deviation of 13.4 years. There were 6 men and 12 women. The majority of the tumors $(72 \%)$ were from the submandibular gland, while 5 tumors (28\%) were from the sublingual gland.

All patients without distant metastasis were treated with curative surgical resection. Patients diagnosed with malignant tumors were offered adjuvant or postoperative radiation. In addition, the 2 patients who presented with distant metastasis (lung, liver and/or lymph nodes) underwent chemotherapy.

FDG PET/CT protocol. Combined PET-CT was performed using a PET-CT scanner (Discovery LS; GE Healthcare) and standard techniques. The patients were fasted for at least $6 \mathrm{~h}$ prior to the examination and their blood glucose level was $<250 \mathrm{mg} / \mathrm{dl}$ (normal, $<200 \mathrm{mg} / \mathrm{dl}$ for non-diabetic and $<250 \mathrm{mg} / \mathrm{dl}$ for diabetic patients). The patients received a barium sulfate oral suspension (Readi-CAT ${ }^{\circledR} 2$; Bracco
Diagnostics Inc.). No intravenous contrast agent was used. Spiral low-dose CT (80 mA, $140 \mathrm{kV}$ and $4 \mathrm{~mm}$ section thickness) was performed in the cranio-caudal direction covering the areas from the vertex to the mid-thigh for the purpose of attenuation correction and anatomic localization. Thereafter, an emission scan was performed in a reverse direction.

MIM imaging software, version 6.4 (MIM Software, Inc.) was used for image display and analysis. The whole-body maximum-pixel intensity projection was used for visual evaluation. The maximum standardized uptake value (SUVmax) of the lesions was recorded. SUVmax is reported to be a more accurate estimate of the true SUV compared with SUVmean (17). For this reason, the use of SUVmax is becoming increasingly more common. Studies have demonstrated that SUVmax is a robust metric for prognosis in certain cancers, and it may also be used to assess response to treatment (17-22).

Image analysis. CT and MRI scans were interpreted by a radiologist who specializes in head and neck imaging. Intravenous contrast material was used in both types of imaging. The scans were analyzed by the extent of the salivary gland lesion, as well as any significant cervical lymph nodes, without any prior knowledge of the clinical findings. Primary salivary lesions were noted on extent, enhancement pattern and location. The regional lymph nodes of the head and neck were also evaluated on the basis of size and SUV value. Both CT and MRI are considered as acceptable methods to be used interchangeably in the evaluation of salivary gland tumors and anatomic imaging. The current literature states that cross-sectional imaging, either CT or MRI, may be used to evaluate salivary gland tumors at the discretion of the attending physician (21). Our sample set included $41 \mathrm{CT}$ and 2 MRI scans from our patients that were used to compare to PET/CT studies. As the $2 \mathrm{MRIs}$ are too small a sample to perform a separate statistical analysis, CT and MRI scans were pooled together.

All PET/CT studies were reviewed by a nuclear medicine physician who is experienced in interpreting head and neck imaging. The images were reviewed without having any knowledge of the clinical findings. The scans were initially reviewed for any abnormal uptake of F-18 FDG in the salivary gland and neck lymph nodes by using the maximal uptake values with higher intensities compared with those of the surrounding tissues. Any abnormal location or asymmetry in the uptake of F-18 FDG was also noted. The interpretation of the nuclear medicine physician was then revised based on the anatomic information of the uptake based on the combined F-18 FDG PET/CT images.

Diagnostic workup. Histopathological and/or cytopathological diagnosis was obtained for all cases of F-18 FDG PET/CT imaging that were included in the present study.

Statistical analysis. For initial lymph node staging and detecting locoregional recurrence, the sensitivity, specificity, positive predictive value (PPV), negative predictive value (NPV), and accuracy of PET/CT, CT or MRI were calculated by comparison with pathological findings and expressed as percentages. The confidence intervals (CIs) for diagnostic 
Table I. Patient characteristics $(n=18)$.

\begin{tabular}{|c|c|}
\hline Characteristics & $\mathrm{n}(\%)$ \\
\hline Age (years), mean (standard deviation) & $58.1(13.4)$ \\
\hline Dose of FDG administered (mCi), mean (standard deviation) & $15.2(2.0)$ \\
\hline Blood glucose level (mg/dl), mean (standard deviation) & $109.4(34.9)$ \\
\hline \multicolumn{2}{|l|}{ Sex } \\
\hline Male & $6(33)$ \\
\hline Female & $12(67)$ \\
\hline \multicolumn{2}{|l|}{ Gland affected } \\
\hline Sublingual & $5(28)$ \\
\hline Submandibular & $13(72)$ \\
\hline \multicolumn{2}{|l|}{ Disease (classification) } \\
\hline Mucoepidermoid carcinoma (malignant) & $5(28)$ \\
\hline Adenoid cystic carcinoma (malignant) & $3(17)$ \\
\hline Lymphoma (malignant) & $3(17)$ \\
\hline Squamous cell carcinoma (malignant) & $1(6)$ \\
\hline Basal cell adenocarcinoma (malignant) & $1(6)$ \\
\hline Submandibular duct carcinoma (malignant) & $1(6)$ \\
\hline Synovial cell sarcoma (malignant) & $1(6)$ \\
\hline Pleomorphic adenoma (benign) & $2(11)$ \\
\hline Lymphoid hyperplasia (benign) & $1(6)$ \\
\hline Total malignant & $15(83)$ \\
\hline Total benign & $3(17)$ \\
\hline \multicolumn{2}{|l|}{ Purpose of PET/CT $(n=43)$} \\
\hline Staging & $14(33)$ \\
\hline Restaging & $29(67)$ \\
\hline
\end{tabular}

FDG, fluorodeoxyglucose; PET, positron emission tomography; CT, computed tomography.

accuracy are 'exact' Clopper-Pearson CIs. P<0.05 was considered to indicate statistically significant differences.

\section{Results}

Patient characteristics. A total of 18 patient cases were analyzed in the present study, including 6 men and 12 women, with a mean age of 58.1 \pm 13.8 years (range, $38-85$ years). The characteristics of the patients included in the study are summarized in Table I. The findings of F-18 FDG PET/CT and CT or MRI were characterized in groups of true positives, false positives, false negatives and true negatives. Detailed information on all the patients considered in the study are included in Table II.

Primary tumor detection. For predicting primary tumors, CT or MRI had a sensitivity of $93.3 \%$, specificity of $75.0 \%$,PPV of $82.4 \%$, and NPV of $90.0 \%$ (Table III). Comparatively, PET/CT scans had a sensitivity of $95.2 \%$, specificity of $100.0 \%$, PPV of $100.0 \%$ and NPV of $95.7 \%$ (Table III). The diagnostic accuracy of $\mathrm{PET} / \mathrm{CT}$ imaging for primary tumor detection was 97.7\% (95\% CI: 87.7-99.9\%), whereas the diagnostic accuracy for CT or MRI for primary tumor detection was $85.2 \%$ (95\% CI: 66.3-95.8\%). Thus, the diagnostic accuracy of both PET/CT and CIS for primary tumor detection was comparable and the difference was not statistically significant.

True negatives included patients who were scanned for staging or restaging purposes. Those patients underwent a CT or MRI within a 6 month time window of their PET/CT imaging. There were 20 true positives for primary tumor detection for PET/CT, whereas CT or MRI scans accurately detected the tumors in 14/20 scans. PET/CT confirmed or identified primary neoplasms, which were all corroborated by subsequent histopathological analysis.

A total of $22 \mathrm{PET} / \mathrm{CT}$ images were true negatives. These cases were follow-ups for restaging. A total of 29 restaging scans were performed. Restaging was performed between 1 and 8 years after treatment by clinical examination and imaging.

There was one false negative case on PET/CT: A case of submandibular adenoid cystic carcinoma, in which the lesion did not display increased SUV; however, the lesion was obvious on CT scan with an additional mass effect (Fig. 1A).

The false positive CT scans for primary tumor included one case with reported enlargement of the lymph nodes, which may be suggestive of malignancy, but a full evaluation was difficult without contrast; and another case of chronic sialadenitis, which was detected on PET/CT but was suggestive of malignancy on CT scan. The false negative CT scan was 


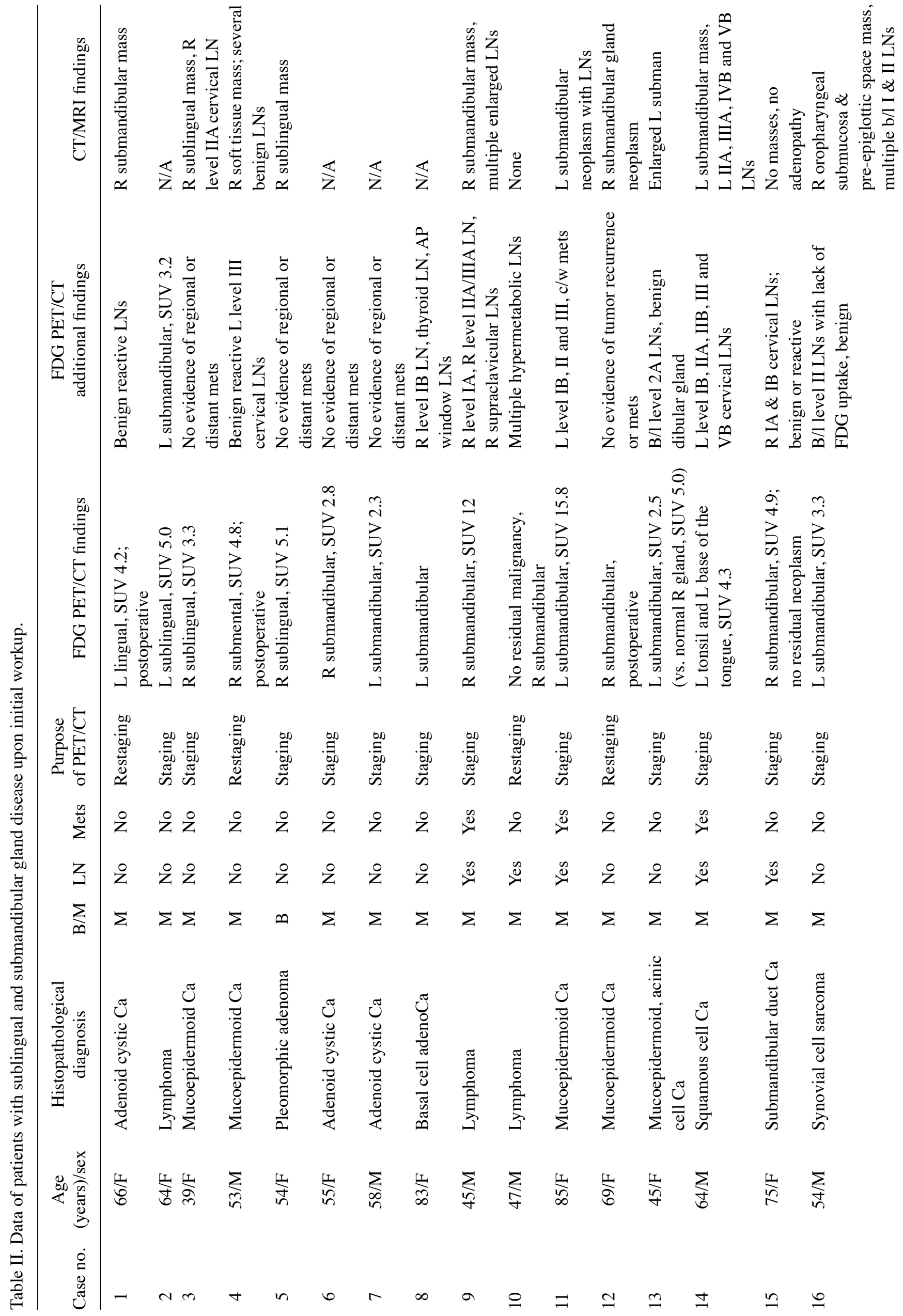


Table III. Comparison of PET/CT and CT/MRI performance for primary tumor detection and cervical lymph node staging.

A, Performance for primary tumor detection

\begin{tabular}{lccccc}
\hline & \multicolumn{2}{c}{ PET/CT } & & \multicolumn{2}{c}{ CT/MRI } \\
\cline { 2 - 3 } \cline { 5 - 6 } Variables & Positive & Negative & & Positive & Negative \\
\hline Pathology & & & & \\
Positive & 20 & 1 & & 14 & 1 \\
Negative & 0 & 22 & 3 & 9 \\
Sensitivity (\%) & 95.2 & & 93.3 & \\
Specificity (\%) & 100.0 & & 75.0 & \\
PPV (\%) & 100.0 & & 82.4 & \\
NPV (\%) & 95.7 & & 90.0 & \\
Accuracy (\%) & 97.7 & & 85.2 & \\
Confidence & $87.7-99.9$ & & $66.3-95.8$ & \\
interval (\%) & & & &
\end{tabular}

B ,Performance for cervical LN staging

\begin{tabular}{|c|c|c|c|c|}
\hline \multirow[b]{2}{*}{ Variables } & \multicolumn{2}{|c|}{$\mathrm{PET} / \mathrm{CT}$} & \multicolumn{2}{|c|}{ CT/MRI } \\
\hline & Positive & Negative & Positive & Negative \\
\hline \multicolumn{5}{|l|}{ Pathology } \\
\hline Positive & 8 & 1 & 6 & 4 \\
\hline Negative & 1 & 33 & 5 & 12 \\
\hline Sensitivity (\%) & 88.9 & & 54.5 & \\
\hline Specificity (\%) & 97.1 & & 75.0 & \\
\hline PPV $(\%)$ & 88.9 & & 60.0 & \\
\hline NPV (\%) & 97.1 & & 70.6 & \\
\hline Accuracy (\%) & 95.4 & & 66.7 & \\
\hline $\begin{array}{l}\text { Confidence } \\
\text { interval }\end{array}$ & $84.2-99.4 \%$ & & $46.0-83.5 \%$ & \\
\hline
\end{tabular}

PET, positron emission tomography; CT, computed tomography; MRI, magnetic resonance imaging; PPV, positive predictive value; NPV, negative predictive value; LN, lymph node.

unable to distinguish between inflammation and invasive submandibular duct carcinoma, whereas PET/CT was able to discriminate between the two.

Cervical lymph node metastasis. For predicting cervical lymph node metastasis, CT or MRI exhibited a sensitivity of $54.5 \%$, specificity of $75.0 \%$, PPV of $60.0 \%$ and NPV of $70.6 \%$ (Table III), whereas PET/CT had a sensitivity of $88.9 \%$, specificity of $97.1 \%$, PPV of $88.9 \%$ and NPV of $97.1 \%$ (Table III). The diagnostic accuracy of PET/CT imaging for detection of lymph node metastasis was $95.4 \%$ (95\% CI: 84.2-99.4\%), whereas the diagnostic accuracy for CT or MRI was $66.7 \%$ (95\% CI: $46.0-83.5 \%$ ). There is a statistically significant difference between PET/CT and CT for the evaluation of cervical lymph node metastasis, as the 95\% confidence intervals do not overlap. PET/CT was able to 

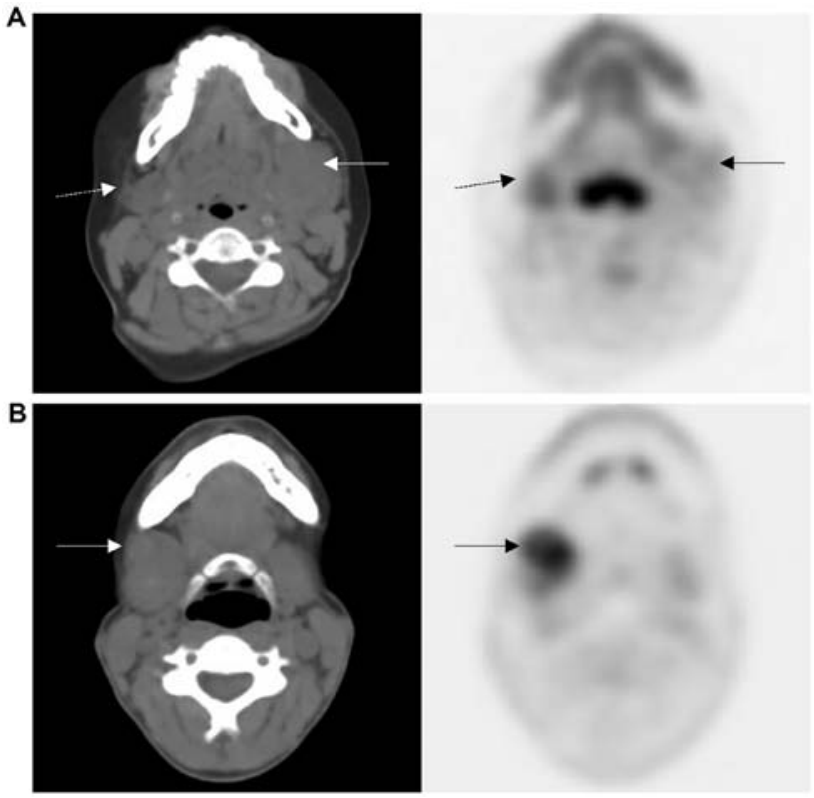

Figure 1. SUVmax is not correlated with malignancy. (A) Axial image of fluorine-18 fluorodeoxyglucose positron emission tomography/computed tomography of a 45 -year-old woman with a history of a left submandibular mass. The mass was later diagnosed as a malignant acinic cell carcinoma of the left submandibular gland with mild uptake (solid arrow, SUV 2.5) Interestingly, the intensity of the tumor SUV uptake is lower compared with that of the contralateral normal right submandibular gland (dashed arrow, SUV 5.0). (B) Axial image of fluorine-18 fluorodeoxyglucose positron emission tomography/computed tomography of a 38-year-old woman with a benign pleomorphic adenoma (arrow), exhibiting a high SUV of 6.9. SUV, standardized uptake value.

detect lymph node metastasis 2 years postoperatively, whereas $\mathrm{CT}$ was unable to detect metastasis during surveillance in a 54-year-old man with synovial cell sarcoma of the left submandibular gland (Fig. 2).

In 9 of the $18(50 \%)$ patients, PET/CT revealed F-18 FDG-avid lymphadenopathy suspicious for metastases to the cervical lymph nodes. These findings would affect treatment options regarding surgical and/or radiation fields.

Distant metastasis. In 4 of the 18 patients of the cohort, the PET/CT findings included avid lymphadenopathy suspicious for metastasis to distant regions. There was 1 case of submandibular synovial cell carcinoma with metastasis to the lung, and 1 case of submandibular squamous cell carcinoma with multiple metastases to the lung and liver (Fig. 3). A table for distant metastases comparing PET/CT vs. CT or MRI was not included, as metastasis was only identified on PET/CT in 4 patients in our sample. No further imaging was performed (in terms of CT or MRI) for distant metastasis to specific regions, such as the liver, lung, or heart in our patients.

Differentiating between benign and malignant tumors. Benign and malignant lesions in our study did not differ significantly in terms of SUVmax. The mean SUVmax for malignant lesions was 5.88, with a standard deviation of 4.25 (Table IV). This may be due to the fact that some benign tumors also have high glucose metabolism. Both false positive cases were pleomorphic adenomas. There were 2 cases of false positive F-18 FDG PET/CT in primary tumor detection. Abnormally
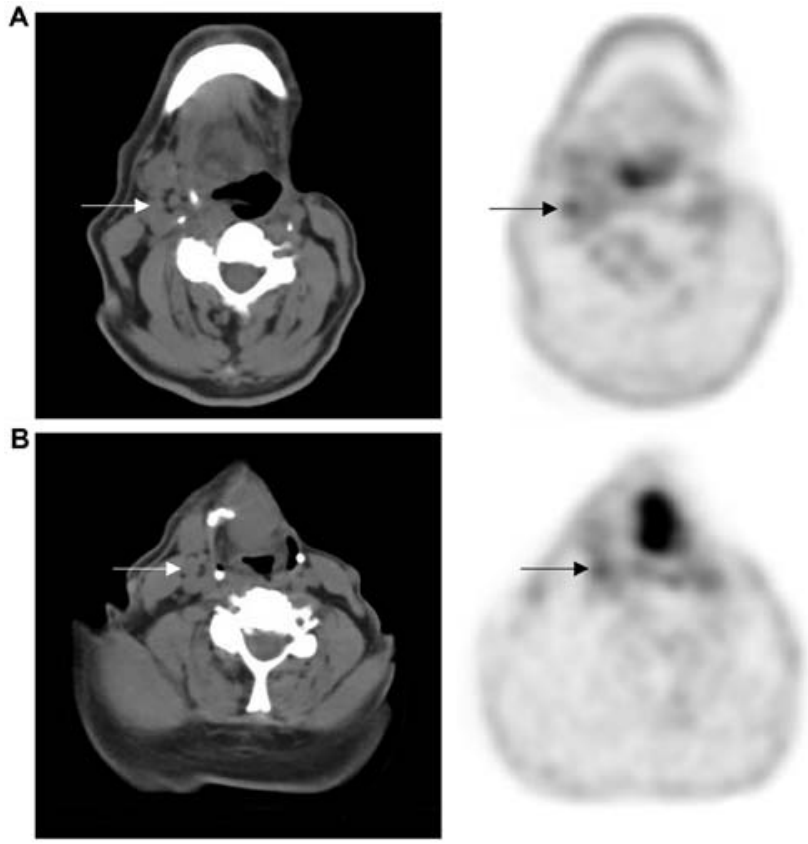

Figure 2. Axial image of fluorine-18 fluorodeoxyglucose PET/CT obtained 2 years postoperatively in a 54-year-old man with a history of T1bNOMx synovial cell sarcoma of the left submandibular gland. (A) This screening $\mathrm{PET} / \mathrm{CT}$ was able to identify nodal metastasis in the (A) right IIA lymph node (arrow) and the (B) right IIIA lymph node (arrow), which was not detected on CT scan. PET, positron emission tomography; CT, computed tomography.

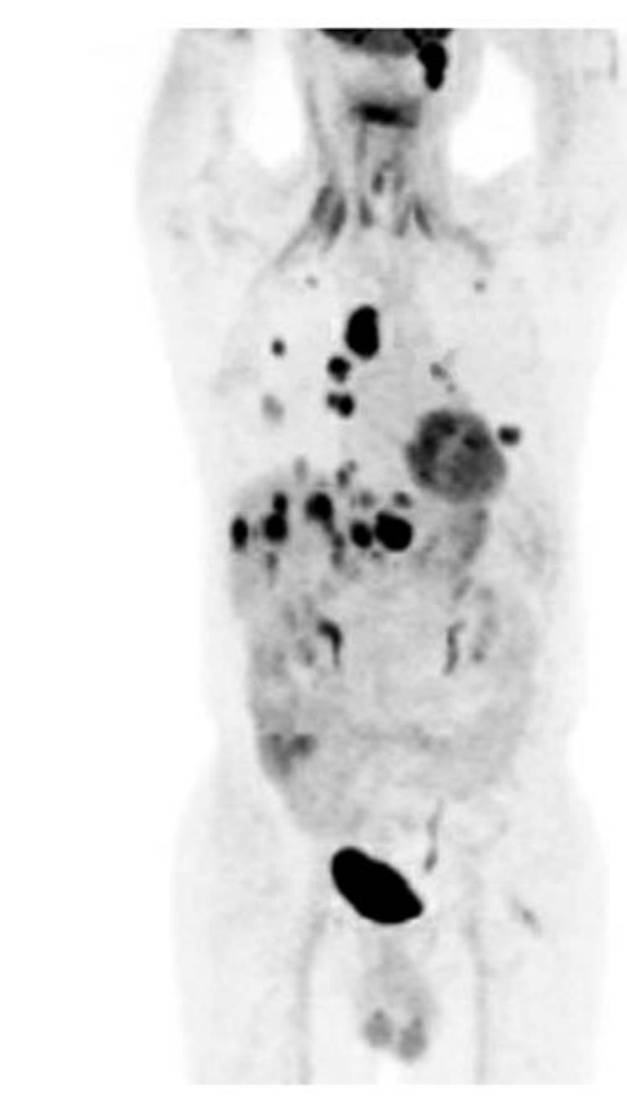

Figure 3. Axial image of fluorine-18 fluorodeoxyglucose positron emission tomography/computed tomography performed for restaging of a 65-year-old man with a history of T1bN3Mx squamous cell carcinoma of the left submandibular gland. The diagnosis of metastatic/recurrent disease was made by the identification of multiple new fluorodeoxyglucose-avid lesions. There was tumor recurrence and multiple metastatic lesions observed in the lungs, mediastinal lymph nodes and liver. 
Table IV. Differentiation between benign and malignant lesions by SUVmax.

\begin{tabular}{lc}
\hline Diagnostic parameters & Values \\
\hline SUVmax in benign vs. malignant & \\
lesions, mean (SD) & $5.88(4.25)$ \\
Malignant & $4(3.58)$ \\
Benign & \\
Performance of SUVmax & $11 / 4$ \\
Malignant, hot/cold (n) & $2 / 1$ \\
Benign, hot/cold (n) & 73.3 \\
Sensitivity (\%) & 33.3 \\
Specificity (\%) & 66.7 \\
Accuracy (\%) & 84.6 \\
Positive predictive value (\%) & 20.0 \\
Negative predictive value $(\%)$ &
\end{tabular}

SUVmax, maximum standardized uptake value; hot, >SUVmax; cold, <SUVmax; SD, standard deviation.

increased F-18 FDG uptake was suspicious of a malignant tumor, but subsequent biopsy and/or surgical resection and pathological examination did not identify malignancy. Both lesions displayed high SUVmax and were biopsied. They were found to be benign, but displayed high uptake of F-18 FDG, and were finally diagnosed as pleomorphic adenoma and benign reactive lymphadenopathy. The scan of a 38 -year-old woman with a $2.5 \times 2.7-\mathrm{cm}$ mass with a SUVmax of 6.9 , which was later pathologically diagnosed as a pleomorphic adenoma after excision, is shown in Fig. 1B.

\section{Discussion}

Submandibular and sublingual salivary gland tumors are rare head and neck tumors that only make up a small fraction of salivary gland tumors. CT or MRI have been widely used for the detection of the primary salivary gland tumors; however, other modalities, such as F-18 FDG PET/CT, are also now being utilized. The aim of the present study was to uncover the role of F-18 FDG PET/CT in the management of submandibular and sublingual salivary gland tumors compared with concurrent CIS, such as CT or MRI. The results revealed that F-18 FDG PET/CT significantly improved the diagnostic accuracy for evaluating the extent of tumor invasion of the lymph nodes and tumor staging compared with CT or MRI alone for patients with submandibular or sublingual salivary gland tumors. The data also revealed that F-18 FDG PET/CT imaging allowed for the evaluation of distant metastasis for such neoplasms. These results are consistent with those of other studies recommending whole-body F-18 FDG PET/CT imaging for detecting distant metastases (22-24). Due to the rare nature of salivary gland tumors, the literature on submandibular and sublingual salivary gland tumors is scarce.

Promising data regarding the prognostic value and detection of metastasis with F-18 FDG PET/CT imaging in salivary gland tumors were recently reported (25). In a systemic review of 22 studies, F-18 FDG-PET/CT was shown to be useful for staging malignant salivary gland tumors, in particular by detecting cervical lymph node and distant metastases in high-grade tumors (26). Although the role of F-18 FDG PET/CT in the initial staging of salivary gland tumors remains controversial, early detection of distant metastases is a unique advantage of this imaging technique. It was recently demonstrated that the use of F-18 FDG PET/CT for staging and restaging provides important information for treatment planning for patients with highly malignant salivary gland tumors (24).

Another goal of the present study was to evaluate the potential of F-18 FDG PET/CT in differentiating between benign and malignant submandibular tumors. The limitation with SUVmax analysis is the heterogeneity of the tumors. Different types of malignancies exhibit different tumor cell metabolic activity. It has been reported that benign Warthin gland tumors and pleomorphic adenomas have high SUVmax (23). Benign conditions, such as infections, obstructive lithiasis, postoperative changes and radiation-induced sialadenitis, may also be associated with increased F-18 FDG uptake in the salivary glands $(23,27,28)$. Asymmetry of uptake is also considered to be suggestive of a possible malignancy, but it may be difficult to distinguish from asymmetrical uptake following surgery and radiation therapy.

Researchers have attempted to elucidate the parameters of PET/CT that may help differentiate between benign and malignant lesions, but without success. Studies on parotid gland tumors have included total lesion glycolysis, metabolic tumor volume, standardized added metabolic activity and normalized standardized added metabolic activity, but without significant differences in salivary gland lesions (29). Other approaches, including dual-time-point F-18 FDG PET/CT, have also been considered, but there has been no conclusive evidence of the differences between benign and malignant salivary gland tumors (30). Therefore, PET/CT scans have not been shown to be useful in discriminating between benign and malignant salivary gland tumors (31). This may be due to the fact that benign tumors, such as Warthin's tumor and pleomorphic adenoma, may also present with high glucose uptake values on imaging due to the increased glucose metabolism (31).

Another difficulty of interpreting salivary gland F-18 FDG PET/CT imaging results from the fact that intense F-18 FDG tracer uptake is normally observed in the palatine tonsils, soft palate and lingual tonsils, while accumulation is variable in the sublingual and submandibular glands (32). It has been noted that the sublingual and submandibular glands exhibit low-to-moderate physiological uptake that varies among patients $(23,32)$. Structures such as the mucosa of the soft and hard palate, major salivary glands, minor salivary glands beneath the mucosa, head and neck muscles, brown adipose tissue, thyroid gland and cerebral cortex naturally display physiological F-18 FDG uptake (23).

However, F-18 FDG PET/CT imaging has the additional advantage that it is a whole-body imaging modality, which is necessary for detecting distant metastases or secondary malignancies. Overall, F-18 FDG PET/CT is sensitive in detecting the primary tumor $(31,33)$ and it is also an accurate modality for staging and restaging malignant salivary gland tumors (15,31). Although F-18 FDG PET/CT demonstrated 
better diagnostic accuracy in the present study, the combined information obtained from F-18 FDG PET/CT and other imaging modalities may enable more accurate characterization of the salivary gland lesion. F-18 FDG PET/CT imaging, therefore, should be used in conjunction with other imaging modalities to verify the results. Biopsy and cytology remain crucial steps in the work-up of sublingual and submandibular salivary gland tumors.

There were certain limitations to the present study: The study was retrospective in nature, with a small patient sample and heterogeneity of cases and primary tumors. Only 18 cases of sublingual or submandibular gland tumors were identified in our high-volume academic institution over an 8-year period, which reflects the rarity of this disease. Due to the retrospective nature, there was little control over the work-up flow of the tumors. There may be selection or referral bias regarding the decision to perform PET/CT, MRI, or CT imaging of the lesions. In addition, the heterogeneity of the tumors may also affect the results. Due to the retrospective nature of the study, the CIS that were analyzed were either CT or MRI rather than a single modality. In addition, no further CT or MRI scans were performed in patients with distant metastasis identified on PET/CT scans. Therefore, this aspect of diagnostic value for CT or MRI was not analyzed in the present study.

Furthermore, there have been correlations between PET/CT SUVmax and histology in other cancers, such as breast and pancreatic cancer $(34,35)$. However, there is a limited number of reported cases of each histological type of malignant salivary gland tumor due to the rarity of malignant sublingual and submandibular salivary gland tumors. In the present study, there were only a few cases of each malignant histological type in our collection of data. Such data evaluation would have a limited power of analysis. Future studies with larger sample sizes may further explore the value of PET/CT SUVmax among different histological types of malignant salivary gland tumors.

In conclusion, the results of the present study indicate that F-18 FDG PET/CT has a higher diagnostic accuracy compared with CIS for evaluating lymph node metastasis and may be useful for screening for distant metastasis ( $\mathrm{N}$ and $\mathbf{M}$ staging) in patients with submandibular or sublingual salivary gland tumors. This non-invasive imaging modality plays an important role in the visualization of the morphology and activity of the tumors, in order to establish a diagnosis, formulate a treatment plan, and initiate possible surgical intervention.

\section{Acknowledgements}

Not applicable.

\section{Funding}

No funding was received.

\section{Availability of data and materials}

The datasets generated and/or analyzed during the present study are available from the corresponding author on reasonable request.

\section{Authors' contributions}

SM and YL were responsible for drafting the article or revising it critically for important intellectual content. SM and YL contributed to the conception and design, and acquisition of data, and analysis and interpretation of data. Both authors have read and approved the final version of this manuscript.

\section{Ethics approval and consent to participate}

The present retrospective study was approved by the Institutional Review Board of Rutgers University (study ID Pro2018001712), and the requirement for written informed consent was waived.

\section{Patient consent for publication}

Not applicable.

\section{Competing interests}

The authors declare that they have no competing interests.

\section{References}

1. Guzzo M, Locati LD, Prott FJ, Gatta G, McGurk M and Licitra L: Major and minor salivary gland tumors. Crit Rev Oncol Hematol 74: 134-148, 2010.

2. Pinkston JA and Cole P: Incidence rates of salivary gland tumors: Results from a population-based study. Otolaryngol Head Neck Surg 120: 834-840, 1999.

3. Han MW, Cho KJ, Roh JL, Choi SH, Nam SY and Kim SY: Patterns of lymph node metastasis and their influence on outcomes in patients with submandibular gland carcinoma. J Surg Oncol 106: 475-480, 2012.

4. Aro K, Ho AS, Luu M, Kim S, Tighiouart M, Clair JM, Yoshida EJ, Shiao SL, Leivo I and Zumsteg ZS: Development of a novel salivary gland cancer lymph node staging system. Cancer 124: 3171-3180, 2018.

5. Ali S, Palmer FL, Yu C, DiLorenzo M, Shah JP, Kattan MW, Patel SG and Ganly I: Postoperative nomograms predictive of survival after surgical management of malignant tumors of the major salivary glands. Ann Surg Oncol 21: 637-642, 2014.

6. Koyuncu M, Sesen T, Akan H, Ismailoglu AA, Tanyeri Y, Tekat A, Unal $R$ and Incesu L: Comparison of computed tomography and magnetic resonance imaging in the diagnosis of parotid tumors. Otolaryngol Head Neck Surg 129: 726-732, 2003.

7. Freling N, Crippa F and Maroldi R: Staging and follow-up of high-grade malignant salivary gland tumours: The role of traditional versus functional imaging approaches-A review. Oral Oncol 60: 157-166, 2016.

8. Rudack C, Jörg S, Kloska S, Stoll W and Thiede O: Neither MRI, CT nor US is superior to diagnose tumors in the salivary glands-An extended case study. Head Face Med 3: 19, 2007.

9. Afzelius P, Nielsen MY, Ewertsen C and Bloch KP: Imaging of the major salivary glands. Clin Physiol Funct Imaging 36: 1-10, 2016.

10. Kessler AT and Bhatt AA: Review of the major and minor salivary glands, Part 2: Neoplasms and tumor-like lesions. J Clin Imaging Sci 8: 48, 2018.

11. Mayerhoefer ME, Prosch H, Beer L, Tamandl D, Beyer T, Hoeller C, Berzaczy D, Raderer M, Preusser M, Hochmair M, et al: PET/MRI versus PET/CT in oncology: A prospective single-center study of 330 examinations focusing on implications for patient management and cost considerations. Eur J Nucl Med Mol Imaging 47: 51-60, 2020.

12. Cicero G, D'angelo T, Racchiusa S, Salamone I, Visalli C, Bottari A, Blandino A and Mazziotti S: Cross-sectional imaging of parotid gland nodules: A brief practical guide. J Clin Imaging Sci 8: 14, 2018. 
13. Liu Y, Li H, Qin L, Huang X, Su M and Han Z: Prognostic factors in malignant sublingual salivary gland tumors. J Oral Maxillofac Surg 75: 1542-1548, 2017.

14. Pfister DG, Spender S, Brizel DM, Burtness B, Busse PM, Caudell JJ, Cmelak AJ, ColevasAD, Dunphy F, Eisele DW, et al: Head and Neck Cancers, Version 2.2014. Clinical Practice Guidelines in Oncology. J Natl Compr Canc Netw 12: 1454-1487, 2014.

15. Sharma P, Jain TK, Singh H, Suman SK, Faizi NA, Kumar R, Bal C, Malhotra A and Kumar R: Utility of (18)F-FDG PET-CT in staging and restaging of patients with malignant salivary gland tumours: A single-institutional experience. Nucl Med Commun 34: 211-219, 2013.

16. Roh JL, Ryu CH, Choi SH, Kim JS, Lee JH, Cho KJ, Nam SY and Kim SY: Clinical utility of 18F-FDG PET for patients with salivary gland malignancies. J Nucl Med 48: 240-246, 2007.

17. Kinahan PE and Fletcher JW: Positron emission tomography-computed tomography standardized uptake values in clinical practice and assessing response to therapy. Semin Ultrasound CT MR 31: 496-505, 2010.

18. Benz MR, Allen-Auerbach MS, Eilber FC, Chen HJ, Dry S, Phelps ME, Czernin J and Weber WA: Combined assessment of metabolic and volumetric changes for assessment of tumor response in patients with soft-tissue sarcomas. J Nucl Med 49: 1579-1584, 2008.

19. Diao W, Tian F and Jia Z: The prognostic value of SUV measuring on primary lesion and ALN by ${ }^{18} \mathrm{~F}-\mathrm{FDG}$ PET or $\mathrm{PET} / \mathrm{CT}$ in patients with breast cancer. Eur J Radiol 105: 1-7, 2018.

20. Cheng NM, Hsieh CE, Liao CT, Ng SH, Wang HM, Fang YD, Chou WC, Lin CY and Yen TC: Prognostic value of tumor heterogeneity and SUVmax of Pretreatment 18F-FDG PET/CT for salivary gland carcinoma with high-risk histology. Clin Nucl Med 44: 351-358, 2019.

21. Thoeny HC: Imaging of salivary gland tumours. Cancer Imaging 7: 52-62, 2007 .

22. Ruhlmann V, Poeppel TD, Veit J, Nagarajah J, Umutlu L, Hoffmann TK, Bockisch A, Herrmann K and Sauerwein W: Diagnostic accuracy of ${ }^{18} \mathrm{~F}-\mathrm{FDG}$ PET/CT and MR imaging in patients with adenoid cystic carcinoma. BMC Cancer 17: 887, 2017.

23. Purohit BS, Ailianou A, Dulguerov N, Becker CD, Ratib O and Becker M: FDG-PET/CT pitfalls in oncological head and neck imaging. Insights Imaging 5: 585-602, 2014.

24. Lee SH, Roh JL, Kim JS, Lee JH, Choi SH, Nam SY and Kim SY: Detection of distant metastasis and prognostic prediction of recurrent salivary gland carcinomas using ${ }^{18} \mathrm{~F}-\mathrm{FDG} \mathrm{PET} / \mathrm{CT}$. Oral Dis 24: 940-947, 2018.
25. Park MJ, Oh JS, Roh JL, Kim JS, Lee JH, Nam SY and Kim SY: 18F-FDG PET/CT Versus Contrast-Enhanced CT for staging and prognostic prediction in patients with salivary gland carcinomas. Clin Nucl Med 42: e149-e156, 2017.

26. Bertagna F, Nicolai P, Maroldi R, Mattavelli D, Bertoli M, Giubbini R, Lombardi D and Treglia G: Diagnostic role of (18) F-FDG-PET or PET/CT in salivary gland tumors: A systematic review. Rev Esp Med Nucl Imagen Mol 34: 295-302, 2015.

27. Bhargava $P$, Rahman $S$ and Wendt J: Atlas of confounding factors in head and neck PET/CT imaging. Clin Nucl Med 36: e20-e29, 2011.

28. Palacios E, Ellis M, Lam EC, Neitzschman H and Haile M: Pitfalls in imaging the submandibular glands with PET/CT. Ear Nose Throat J 94: E37-E39, 2015.

29. Kendi AT, Magliocca KR, Corey A, Galt JR, Switchenko J, Wadsworth JT, El-Deiry MW, Schuster DM, Saba NF and Hudgins PA: Is there a role for PET/CT parameters to characterize benign, malignant, and metastatic parotid tumors? AJR Am J Roentgenol 207: 635-640, 2016.

30. Toriihara A, Nakamura S, Kubota K, Makino T, Okochi K and Shibuya H: Can dual-time-point 18F-FDG PET/CT differentiate malignant salivary gland tumors from benign tumors? AJR Am J Roentgenol 201: 639-644, 2013.

31. Park HL, Yoo Ie R, Lee N, Yoon H, Choi EK, Choi HS and Kim SH: The value of F-18 FDG PET for planning treatment and detecting recurrence in malignant salivary gland tumors: Comparison with conventional imaging studies. Nucl Med Mol Imaging 47: 242-248, 2013.

32. Nakamoto Y, Tatsumi M, Hammoud D, Cohade C, Osman MM and Wahl RL: Normal FDG distribution patterns in the head and neck: PET/CT evaluation. Radiology 234: 879-885, 2005

33. Kim JY, Lee SW, Kim JS, Kim SY, Nam SY, Choi SH, Roh JL, Choi EK, Kim JH, Song SY, et al: Diagnostic value of neck node status using 18F-FDG PET for salivary duct carcinoma of the major salivary glands. J Nucl Med 53: 881-886, 2012.

34. Bitencourt AG, Lima EN, Chojniak R, Marques EF, de Souza JA, Graziano L, Andrade WP and Osório CA: Correlation between PET/CT results and histological and immunohistochemical findings in breast carcinomas. Radiol Bras 47: 67-73, 2014.

35. Myssayev A, Myssayev A, Ideguchi R, Eguchi S, Adachi T, Sumida Y, Tobinaga S, Uetani M and Kudo T: Usefulness of FDG PET/CT derived parameters in prediction of histopathological finding during the surgery in patients with pancreatic adenocarcinoma. PLoS One 14: e0210178, 2019. 